\title{
Intraperitoneal chemotherapy for peritoneal metastases: confronting diversity, maximizing benefit
}

As Kurt Van der Speeten and Paul Sugarbaker accepted the responsibility to edit this Focused Issue of Fournal of Gastrointestinal Oncology (7GO), we committed ourselves to expand the manuscripts with an additional layer of critical commentary regarding the efficacy of intraperitoneal (IP) chemotherapy for peritoneal metastases (PM). One should know that at the very beginning our authors were repeatedly admonished to lead the reader to the best HIPEC, EPIC, NIPEC or PIPAC that was currently available for their particular disease process. Over and above this charge to our authors, the Editors have attempted to preface this focused issue with a critical evaluation of its contents. Our special role as Editors was to critically evaluate the state-of-the-art and science of IP chemotherapy for PM as presented in this JGO Focused Issue and recommend the next steps in the way forward.

Two observations regarding the treatment of PM in general emerged during the last decade. First, the rush of optimism regarding IP chemotherapy for PM from invasive cancers was premature. Yes, there is an improved outcome for mucinous appendiceal neoplasms and epithelial malignant peritoneal mesothelioma that is truly spectacular! By analogy, many of us immersed in peritoneal surface malignancy (PSM) assumed that the new treatments would result in much more favorable outcomes for peritoneal metastases from gastric cancer, colorectal cancer, ovarian cancer, and even pancreas malignancy. Great progress in the surgical treatment options for PM have occurred. The outcome is always better if a complete cytoreduction is possible. However, we have all had to accept the fact that small increments of benefit are to be expected when currently available intraperitoneal chemotherapy regimens are used for these invasive peritoneal malignancies. Breakthroughs are not to be expected and randomized controlled trials are essential to demonstrate limited benefit.

Secondly, we are convinced that IP chemotherapy is being asked to perform over and above that which translational science would predict. IP chemotherapy in an aqueous solution with or without heat enters tissues by simple diffusion. This results in drug penetration of a fraction of a millimeter into cancerous tissue. Also, if the cancer nodule is vascularized, the chemotherapy is rapidly removed from tumor into the body compartment. Consequently, with HIPEC, only single cells or minute nodules should be expected to develop apoptosis. Perhaps only non-vascularized nodules are eradicated. This response assumes that a CC- 0 cytoreduction precedes HIPEC in an invasive epithelial cancer. CC-1 cytoreductions with cancer nodules up to $2.5 \mathrm{~mm}$ may be eradicated by perioperative IP chemotherapy in pseudomyxoma peritonei or malignant peritoneal mesothelioma but not with an invasive malignancy. Not only is the chemotherapy penetration and retention within a PM severely restricted, the chemotherapy that has entered the cell has a low response rate.

For example, mitomycin $\mathrm{C}$ has a durable response rate of approximately $20 \%$ for previously untreated metastatic colon cancer. Add a low volume of disease as expected after complete cytoreduction, increased drug concentration from intraperitoneal drug administration, and heat. The durable response may reach $40 \%$. No surprising, then, that $40 \%$ is the long-term survival expected with cytoreductive surgery and HIPEC for colon cancer.

Unfortunately, even with optimal delivery of chemotherapy into the PM, apoptosis may not result because the cancer cells have natural or acquired resistance. Yet, we wonder why IP chemotherapy is not more effective. In order to maximize its benefit, one needs the cytoreduction to be CC-0 (no visible residual disease), prolonged exposure of the IP chemotherapy and cytotoxic agents active in a majority of patients. Also, valuable lessons are to be learned from the successes of systemic chemotherapy in the last 30 years: progress will be the result of multicycle and multidrug regimens that are founded in extensive translational science (both in-vitro and in-vivo). In the Editors' opinion, these challenges require unrelenting persistence and determination.

Based on the preponderance of evidence, clinical information and impressions, and also from the fact that a majority of peritoneal surface malignancy centers are unwilling to abandon HIPEC, the Editors of this JGO Focused Issue have accepted the challenge to suggest some guidelines for the current and future use of IP chemotherapy. A guideline can streamline a particular practice according to a set routine or sound practice. It is import to be aware that new information may require guidelines to be modified, changed or abandoned. Clinical practice guidelines are usually formulated by experts who have recently published peer-reviewed data. The Editors suggest the following next steps in the way forward for the use of 
intraperitoneal chemotherapy:

(I) All guidelines must be well supported by pharmacologic data.

(II) HIPEC is considered an essential part of all PSM treatments but, in most instances, not sufficient in and of itself. Drugs to be considered for HIPEC are cisplatin, oxaliplatin, mitomycin C, docetaxel, gemcitabine, melphalan, and doxorubicin.

(III) EPIC is associated with clinical and logistical problems but allows cell cycle-specific drugs to be used as perioperative chemotherapy and incorporates the important principle of multicycle drug delivery. Drugs to be considered for EPIC include paclitaxel, 5-fluorouracil and pegylated liposomal doxorubicin.

(IV) NIPEC long-term is administered through an IP port or intraperitoneal catheter. After an extensive cytoreduction often required by ovarian, appendiceal or colorectal cancer, the intraperitoneal ports should only be implanted by very experienced and committed PSM groups. In patients who have had limited visceral resections or no prior surgery, NIPEC through a port is readily accomplished (1). NIPEC is usually combined with intravenous and/or oral chemotherapy. Drugs to be considered for NIPEC long-term are paclitaxel, docetaxel, pemetrexed, gemcitabine, pegylated liposomal doxorubicin and 5-fluorouracil. The only drugs consistently shown to be of benefit are the taxanes. A technical innovation to facilitate long-term IP access would be of great benefit. With reliable long-term access an RCT in malignant peritoneal mesothelioma patients of HIPEC versus HIPEC plus EPIC and NIPEC paclitaxel is recommended. Pegylated liposomal doxorubicin has, from a pharmacologic perspective, great potential. It is in need of a phase I/II trial. A great place to start would be endometrial cancer patients with a high risk for peritoneal progression.

(V) For both gastric cancer with PM and ovarian cancer with PM, a HIPEC regimen of cisplatin and a taxane can be strongly recommended as an optimal intraperitoneal chemotherapy doublet (2-4). The dose of cisplatin is $75 \mathrm{mg} / \mathrm{m}^{2}$ in fit patients. Less fit patients and those with limited bone marrow reserve due to extensive prior chemotherapy would be treated with $50 \mathrm{mg} / \mathrm{m}^{2}$. Docetaxel is used at $30 \mathrm{mg} / \mathrm{m}^{2}$ or paclitaxel at $175 \mathrm{mg} / \mathrm{m}^{2}$. The temperature should be kept at $43{ }^{\circ} \mathrm{C}$ either by open or closed HIPEC methodology. An alternative for both gastric and ovarian malignancy is single agent cisplatin at $100 \mathrm{mg} / \mathrm{m}^{2}$ plus thiosulfate. However, when thiosulfate is used the Editors would like to see pharmacokinetic information about the extent of free platinum available within the peritoneal space for the duration of HIPEC. In these two diseases, RCTs of high dose cisplatin with thiosulfate rescue versus a multidrug regimen with low dose cisplatin is an obvious next step.

(VI) For colorectal and high-grade appendiceal malignancy, HIPEC as a perioperative FOLFOX which includes a single dose of EPIC 5-fluorouracil is recommended (5). In colorectal cancer patients who have had neoadjuvant chemotherapy with FOLFOX, HIPEC with Dutch triple-dose mitomycin C is likely to be most effective. An RCT in colorectal and high grade appendiceal PM is a rational next step. Few peritoneal surface malignancy centers are willing to take on colorectal PM in the absence of HIPEC. Also, there was sufficient "positiveness" in PRODIGE 7 to avoid a cytoreduction only arm of a trial. An RCT of perioperative FOLFOX versus HIPEC with mitomycin C is recommended (5).

(VII) The diversity that currently exists regarding the administration of perioperative chemotherapy needs to be reduced. This Focused Issue of JGO combats this diversity to allow the initiation of randomized trials looking at safety, efficacy and quality of life of one regimen versus another. Single institution trials are appropriate so that confounding variables regarding the technology and methodology of cytoreduction and HIPEC are minimized.

It would be advantageous if a molecular approach to drug selection or other molecular treatment options were available at this point in time (6). However, at the present time for the majority of the conditions we see in the treatment of PM this type of selection is not possible. Hopefully, it will arrive soon but, at this point in time, combinations of HIPEC, EPIC, NIPEC and PIPAC are indicated.

The editors share the view that IP chemotherapy in PM is coming of age. The underlying principle of dose intensification of chemotherapeutic agents in the peritoneal cavity is still sound and of great potential benefit to PM patients. Fortunately, enthusiasm is making place for science. The above mentioned mixed clinical results have stimulated a return to the translational and clinical workbench as demonstrated by the increasing number of recent publications in this field. The result, without doubt, will be more standardized and scientifically validated IP regimens and methodologies for IP application that 
will shortly find their way to testing in RCTs. In the meantime; the IP regimens and results presented by the authors in this issue can guide PSM centers worldwide towards the best current clinical practice.

Respectfully submitted,

The Editors

\section{Acknowledgments}

Funding: None.

\section{Footnote}

Provenance and Peer Review: This article was commissioned by the editorial office, Fournal of Gastrointestinal Oncology for the focused issue "Intraperitoneal Chemotherapy for Peritoneal Metastases: HIPEC, EPIC, NIPEC, PIPAC and More". The article did not undergo external peer review.

Conflicts of Interest: Both authors have completed the ICMJE uniform disclosure form (available at http://dx.doi.org/10.21037/ jgo-2020-07). The focused issue was sponsored by the Peritoneal Surface Oncology Group International (PSOGI). Drs. PHS and KVDS served as the unpaid Guest Editors of the focused issue. The authors have no other conflicts of interest to declare.

Ethical Statement: The authors are accountable for all aspects of the work in ensuring that questions related to the accuracy or integrity of any part of the work are appropriately investigated and resolved.

Open Access Statement: This is an Open Access article distributed in accordance with the Creative Commons AttributionNonCommercial-NoDerivs 4.0 International License (CC BY-NC-ND 4.0), which permits the non-commercial replication and distribution of the article with the strict proviso that no changes or edits are made and the original work is properly cited (including links to both the formal publication through the relevant DOI and the license). See: https://creativecommons.org/licenses/by-nc$\mathrm{nd} / 4.0 \%$.

\section{References}

1. Canbay E, Canbay Torun B, Cosarcan K, et al. Surgery with hyperthermic intraperitoneal chemotherapy after response to induction chemotherapy in patients with peritoneal metastasis of gastric cancer. J Gastrointest Oncol 2021;12:S47-56.

2. Boerner T, Piso P. A narrative review of intraperitoneal chemotherapy and cytoreductive surgery (CRS) for peritoneal metastases in gastric cancer. J Gastrointest Oncol 2021;12:S59-67.

3. Ji ZH, Zhang Y, Li Y. Intra-operative hyperthermic intraperitoneal chemotherapy for prevention and treatment of peritoneal metastases from gastric cancer: a narrative review. J Gastrointest Oncol 2021;12:S70-8.

4. Coccolini F, Fugazzola P, Montori G, et al. Intraperitoneal chemotherapy for ovarian cancer with peritoneal metastases, systematic review of the literature and focused personal experience. J Gastrointest Oncol 2021;12:S144-81.

5. Cashin PH, Graf W. Sequential postoperative intraperitoneal chemotherapy for colorectal cancer with peritoneal metastases: a narrative review. J Gastrointest Oncol 2021;12:S131-5.

6. Lund-Andersen C, Torgunrud A, Fleten KG, et al. Omics analyses in peritoneal metastasis-utility in the management of peritoneal metastases from colorectal cancer and pseudomyxoma peritonei: a narrative review. J Gastrointest Oncol 2021;12:S191-203. 


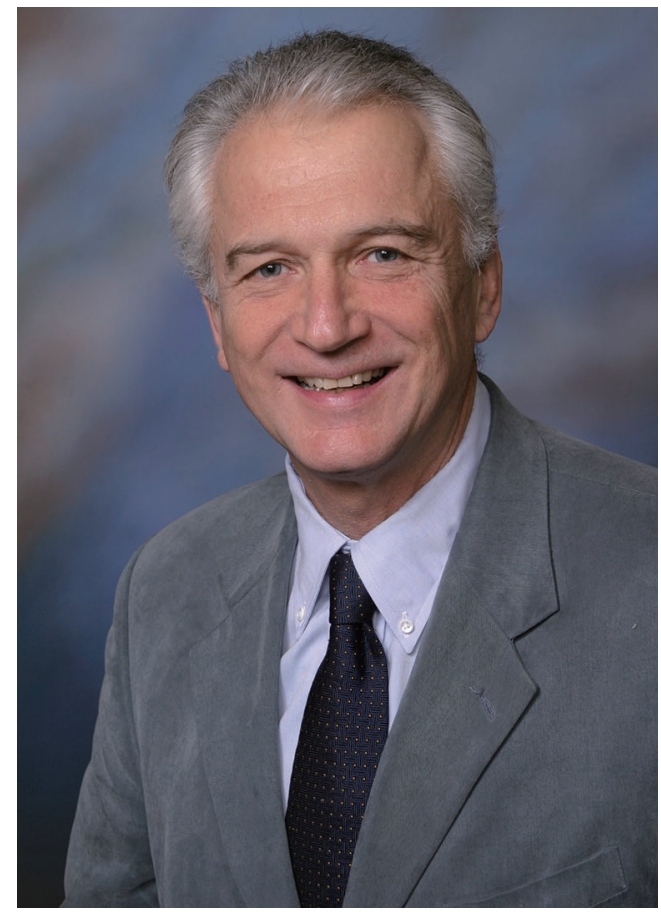

Paul H. Sugarbaker

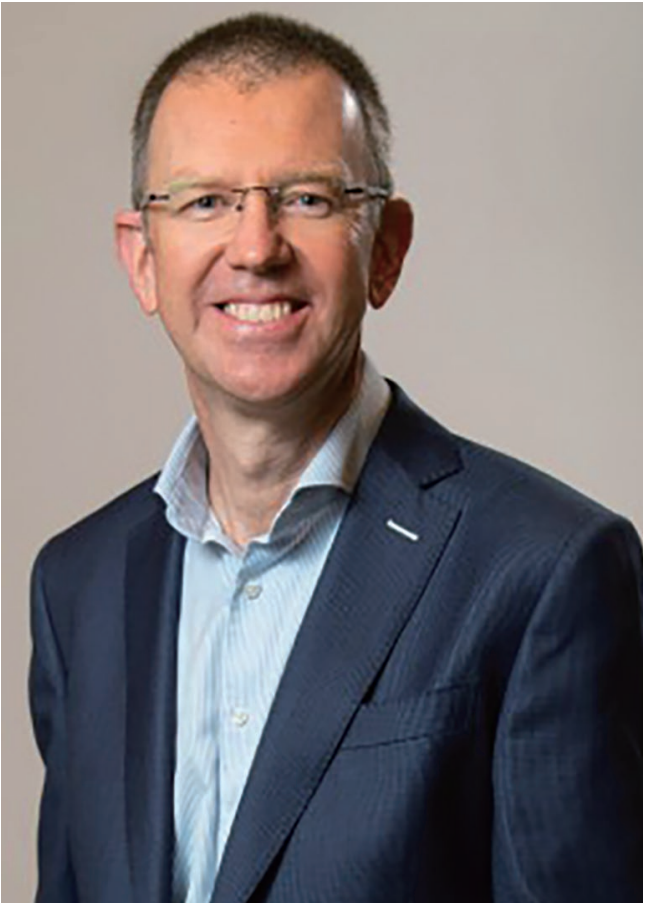

Kurt Van der Speeten

Paul H. Sugarbaker Center for Gastrointestinal Malignancies, MedStar Washington Hospital Center, Washington, DC, USA.

(Email: Paul.Sugarbaker@outlook.com)

Kurt Van der Speeten

Department of Surgery, Hospital Oost-Limburg, Genk, Belgium.

(Email: Kurt.Vanderspeeten@zol.be)

Submitted Nov 26, 2020. Accepted for publication Jan 30, 2021.

doi: 10.21037/jgo-2020-07

View this article at: http://dx.doi.org/10.21037/jgo-2020-07

Cite this article as: Sugarbaker PH, Van der Speeten K. Intraperitoneal chemotherapy for peritoneal metastases: confronting diversity, maximizing benefit. J Gastrointest Oncol 2021;12(Suppl 1):S1-S4. doi: 10.21037/jgo-2020-07 